\title{
Thrive Health: a New Venture Connecting Patient Advocacy to Well-Being
}

\author{
Farina Fassihi $^{1}$
}

Published online: 12 May 2020

(C) Springer Nature Switzerland AG 2020

\begin{abstract}
As part of the British Columbia government's response to the COVID-19 pandemic outbreak, an online self-assessment tool was launched to help with symptom assessment and to provide necessary health information for people. The tool was developed by Thrive Health, a company which has been working to improve the delivery of e-health in Canada since 2016, with an emphasis on well-being. This review captures this company's purpose and role in addressing the needs of patients and healthcare providers in the community, to change the definition of healthcare. In pursuing this goal, Thrive Health is innovating to make the long lost connection between physical and mental health through an online app with design features supported by research around subjective well-being, as a measurement of effective community development and human progress. This very characteristic is what establishes the company's uniqueness among other similar patient advocacy groups, and connects patient advocacy to happiness and well-being.
\end{abstract}

Keywords Thrive Health $\cdot$ Health $\cdot$ Subjective well-being $\cdot$ Patient advocacy

\section{Background}

With the global outbreak of the COVID-19 pandemic came an immediate anxiety. Testing was not immediately available for all; yet people wanted to know whether or not they had been infected. This fear disrupted the work of care clinics and hospitals, as they rushed to serve people in need of urgent care and were also faced with those who demanded testing. While people cannot be blamed for their illness anxiety, the burden they pose has consequences.

As part of the British Columbia government's response to the Coronavirus pandemic, a self-assessment tool was developed on-the-fly, helping people understand their symptoms, and act according to the best available guidance. Updated with the most

Farina Fassihi

farina_fassihi@sfu.ca

1 Urban Studies Program, Simon Fraser University, Vancouver, Canada 
recent guidelines as they have become available, the tool asks six simple questions about symptoms, recent travel history, as well as close contacts. Based on the answers, it advises when to call 911 and take emergency care, when to call 811 to speak to a nurse for further testing and instructions, when to isolate at home, or when to selfmonitor. The tool, used by millions within a few short weeks, has served to calm anxieties and reduce the burden on call centres, clinics and hospitals. The app was developed and deployed within days of the COVID-19 pandemic by a new company called Thrive Health.

This review presents the origins of Thrive Health, which has been working to improve the delivery of healthcare in Canada since 2016. The COVID-19 self-assessment tool provides a useful snapshot of the company's utility and purpose; responding quickly to the real-time needs of the health care professions and patients alike, along with the community as a whole, engaging and assisting these groups based on their need and abilities in pursuit of a new definition of health care that is aligned with well-being.

The story of patients getting lost on their healthcare journey is familiar to almost everybody. When accident or illness strikes, managing one's healthcare can be overwhelming and complicated. This is specifically true for seniors and vulnerable groups. Recognition of this need and inequity has given rise to the move toward patient advocacy, defined in broad strokes as any activity which ultimately benefits a patient. Having an advocate or advocacy group by one's side to help navigate the health care system and process can make all the difference in successful patient recovery and even the efficiency of the health care system. Patient advocacy groups support and promote the rights of the patient, enhance the delivery of quality care, and help build capacity to improve community health on the whole.

For David and James Helliwell, brothers, the idea for Thrive Health emerged from personal experience. Despite different lifestyle and professions - David is a software entrepreneur and James, a cardiothoracic anesthesiologist - they have shared the overwhelming experience of seeking healthcare, along with most of today's adult population. Their experiences have included: frustrations of misdiagnosis, details getting lost with specialist care, jumping from one doctor to the other without the necessary information being shared, lack of timely interventions and updates about their health status. Taking these frustrations as fodder, David and James were inspired to use their combined expertise to build a system that would address existing issues in healthcare systems and bridge certain gaps in diagnosis, treatment and recovery. The result is a smart patient advocacy system, a new company and digital tool, called Thrive Health.

Thrive Health offers a reliable, secure application through which patients can effectively communicate with their medical providers, be updated about their care process and benefit from a smoother, more efficient healthcare experience, in which they feel more informed, more consulted, more in control. By creating an effective method of organizing care records, Thrive Health enables users to track trends in ways that make sense to them, and track changes in their diagnosis, treatment and recovery plans and processes. Servicing approximately 15,000 patients in clinics in Ontario and BC, including Saint Paul's Hospital and BC Children's Hospital, Thrive Health focuses on visibility and sharing of data, in the interest of more informed choices by patients and care providers alike. The app helps people through their health journey by focusing on two stages of a medical problem: preparation (for surgery, labour, etc.) and follow up (the recovery process). 
Thrive Health stands out among similar internet application-based patient advocacy and engagement systems in the way that it integrates a focus on measuring and enhancing happiness and well-being for both patients and care providers into the work of patient advocacy. The goal is no less than that of "changing the definition of what healthcare looks like."

To understand this unique integrative characteristic, one needs to be introduced to another member of the Helliwell family, David and James's father John. John Helliwell, a respected authority in the field of subjective well-being, is Professor Emeritus of Economics at the University of British Columbia, and also editor of the World Happiness Report, Senior Fellow of the Canadian Institute for Advanced Research (CIFAR), and CoDirector of the CIFAR Programme on Social Interactions, Identity, and Well-being. Specifically, Helliwell's research positioning subjective well-being as a measurement of effective community development and human progress is a major inspiration behind Thrive Health. This inspiration gives Thrive Health the promise of finding meaningful ways to connect the treatment of physical and mental health. Seminal research by House et al. (1988) and many more has shown that depression, loneliness, a lack of social connections, and other mental health conditions can have unacknowledged, serious negative effects on physical health - reducing immunity and longevity, among other risks. At Thrive Health, the challenge that the company takes most seriously is to connect physical and mental health status throughout the health care journey, and to open up this discussion between patients and their health care providers.

\section{Key Focus Areas}

\section{Patient Engagement}

One would be excused for thinking that the healthcare system was actually designed to make it hard for patients to focus, prioritize and act in the interest of their own health and wellbeing. Patient advocates are clearly part of the solution. One of the most important factors in effective patient advocacy is the recognition that patients themselves have the "greatest vested interest" in their own health; and with this interest should come influence. Patient engagement could be considered a required skill set for patient advocates to ensure better outcomes for patients. It is of great importance for patients and their families to actively participate in their care as it will lead to a higher level of patient satisfaction (Chang 2017).

Thrive Health is seeking to change a medical model of care to a person-oriented one, and is moving toward providing care "with" people, rather than "for" them. It speaks to a paradigm shift from focusing on illness to promoting wellness. The key to the way this online app works is that most of the information shared is provided by patients themselves, and as a result, patients are no longer passive stakeholders.

\section{A Thriving Score for Well-Being}

Although the fundamental connection between physical and mental health is evident to scholars of wellbeing, in healthcare settings, there remains a separation between the two. At Thrive Health, by contrast, this connection is a two-way street for health care practitioners and patients alike. If mental health is neglected, deteriorating mental 
health can impede physical healing; at the same time, the health care journey itself can have profound impacts on mental health. Recent research published by the Canadian Mental Health Association (2019) found that: "People living with mental illnesses experience a range of physical symptoms that result both from the illness itself and as a consequence of treatment. These people are also at higher risk of experiencing a wide range of physical conditions." Recognizing the links between mind and body is the first step toward strategies to improve patient outcomes. Thrive Health attempts to bridge this existing divide by measuring well-being as a key factor in patient resilience, and integrating such information with the delivery of healthcare. In specifically designed questions, the app uses standard well-being questions, involving subjective well-being, as metrics to get a "Thriving Score" for each patient.

\section{The Enhance Project}

Subjective well-being can influence physical health. Scores of studies show that our levels of happiness versus stress and depression can influence cardiovascular health, immune system strength, and our ability to heal from injuries (Diener et al. 2017). One important figure in this research from which Thrive Health derives new inspiration is Ed Diener, Professor of Psychology at the University of Virginia and the University of Utah, and a Senior Scientist for Gallup. Alongside him is Dr. Carol Diener, who has dedicated her own research to identifying factors that predict subjective well-being. The Enhance programme is a 12-week interactive course that delivers daily exercises to help patients form healthy habits and make better lifestyle choices. As Thrive seeks to highlight connections between mental and physical health, Enhance is a tool with which patients can work toward happiness and wellbeing as an integral part of recovery from illness and injury.

In each of these apps, related to rapid self-assessment and information sourcing for COVID-19, preparing for procedures and recovery, getting a Thriving Score or challenging yourself with the Enhance programme, Thrive Health is innovating at a rapid pace in pursuit of a goal that patients and clinicians connect mental well-being to physical health.

\section{References}

Canadian Mental Health Association. (2019). The relationship between mental health, mental illness and chronic physical conditions. Canadian Mental Health Association. Accessed November 20 ${ }^{\text {th }}, 2019$, https:/ontario.cmha.ca/documents/the-relationship-between-mental-health-mental-illness-and-chronicphysical-conditions/.

Chang, S. (2017). The hidden truth of patient engagement. NEJM Catalyst. https://doi.org/10.1056 /CAT.17.0482.

Diener, E., Pressman, S. D., Hunter, J., \& Delgadillo-Chase, D. (2017). If, why, and when subjective wellbeing influences health, and future needed research. Applied Psychology: Health and Well-Being, 9(2), $133-167$.

House, J. S., Landis, K. R., \& Umberson, D. (1988). Social relationships and health. Science, 241(4865), 540545 .

Publisher's Note Springer Nature remains neutral with regard to jurisdictional claims in published maps and institutional affiliations. 